\title{
An inverse transformation for quadrilateral isoparametric elements: Analysis and application
}

\author{
Chongyu Hua \\ Department of Civil Engineering. The University of Michigan, Ann Arbor, MI 48109, U.S.A.
}

\begin{abstract}
The coordinate transformation for quadrilateral isoparametric elements is well-defined in the finite element literature. However, a corresponding inverse transformation is not found. In fact, it has been commonly believed that no explicit solutions to the inverse transformation problem exist. This paper shows that if geometric considerations are used, a complete set of general solutions to the inverse transformation problem can be derived. An application of the inverse transformation for slope stability analysis is also presented.
\end{abstract}

\section{Introduction}

Two types of coordinate systems are used in finite element analysis: a global coordinate system $(x, y)$ located in a finite element mesh, or element domain $\Omega_{\mathrm{e}}$ and a natural coordinate system $(\xi, \eta)$ located in every master element, or parent domain $\bar{\Omega}_{\mathrm{e}}$. There are primarily two reasons for choosing natural coordinates in addition to global coordinates: one is the ease of constructing trial functions for elements, and the other is the ease of integration within the elements.

The two coordinate systems are related through well-defined mappings. The following expression,

$$
(x, y):\left[(\xi, \eta) \in \bar{\Omega}_{\mathrm{e}}\right] \rightarrow \Omega_{\mathrm{e}},
$$

states that for a given point $(\xi, \eta)$ in the parent domain $\bar{\Omega}_{\mathrm{e}}$, there exists a mapping $(x, y)$ in the element domain $\Omega_{\mathrm{e}}$. In other words, for any given point $(\xi, \eta)$, a mapping is defined by

$$
x=x(\xi, \eta), \quad y=y(\xi, \eta) .
$$

This is commonly referred to as coordinate transformation.

An inverse transformation defines mapping of a point in the element domain $\Omega_{\mathrm{e}}$ back into the parent domain $\bar{\Omega}_{\mathrm{e}}$ in the form

$$
(\xi, \eta):\left[(x, y) \in \Omega_{\mathrm{e}}\right] \rightarrow \bar{\Omega}_{\mathrm{e}}
$$

or

$$
\xi=\xi(x, y), \quad \eta=\eta(x, y) .
$$

A typical quadrilateral isoparametric element in the two coordinate systems is shown in Fig. 1. The two systems are related by the following coordinate transformation:

$$
x(\xi, \eta)=\sum_{i=1}^{4} N_{i}(\xi, \eta) x_{i}, \quad y(\xi, \eta)=\sum_{i=1}^{4} N_{i}(\xi, \eta) y_{i},
$$




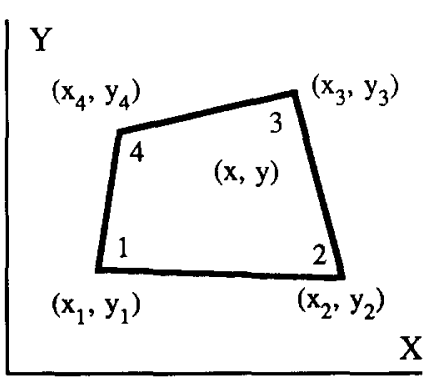

Global Coordinate System

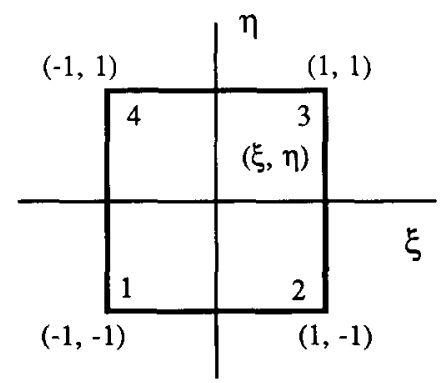

Natural Coordinate System

Fig. 1. A quadrilateral isoparametric element in two coordinate systems.

where the $\left(x_{i}, y_{i}\right)$ 's are global coordinates of nodal points, and the $N_{i}(\xi, \eta)$ 's are shape functions given by

$$
\begin{array}{ll}
N_{1}(\xi, \eta)=\frac{1}{4}(1-\xi)(1-\eta), & N_{2}(\xi, \eta)=\frac{1}{4}(1+\xi)(1-\eta) \\
N_{3}(\xi, \eta)=\frac{1}{4}(1+\xi)(1+\eta), & N_{4}(\xi, \eta)=\frac{1}{4}(1-\xi)(1+\eta)
\end{array}
$$

The coordinate transformation in equation (5) was introduced by Taig [6] in 1961. However, a corresponding inverse transformation is generally not considered in the finite element literature. In fact, it has been commonly believed that no explicit solutions to the inverse transformation problem exist [7]. Although the parent domain $\bar{\Omega}_{\mathrm{e}}$ can generally provide an adequate frame of reference for stress and strain evaluation in most finite element applications, there are some circumstances, such as slope stability analysis, in which the stress or strain evaluation in the element domain $\Omega_{\mathrm{e}}$ becomes necessary. In these circumstances an inverse transformation method is the most efficient approach. This paper will show that if geometric considerations are used, a complete set of general solutions to the inverse transformation problem can in fact be derived. An application of this inverse transformation for slope stability analysis is also presented.

\section{Inverse transformation analysis}

The relationship between the two coordinate systems for quadrilateral isoparametric elements can be written in a general form as

$$
\left[\begin{array}{ll}
b_{1} & c_{1} \\
b_{2} & c_{2}
\end{array}\right]\left\{\begin{array}{l}
\xi \\
\eta
\end{array}\right\}=\left\{\begin{array}{l}
d_{1}-a_{1} \xi \eta \\
d_{2}-a_{2} \xi \eta
\end{array}\right\}
$$

where

$$
\begin{aligned}
& d_{1}=4 x-\left(x_{1}+x_{2}+x_{3}+x_{4}\right), \\
& d_{2}=4 y-\left(y_{1}+y_{2}+y_{3}+y_{4}\right),
\end{aligned}
$$

and the $a$ 's, $b$ 's and $c$ 's are functions of global coordinates of nodal points and depend upon local node numbering schemes. The local node numbering possibilities for a quadrilateral isoparametric element in a finite element mesh are shown in Fig. 2. The coefficients in equation (7) can be obtained by the following equations:

$$
\left[\begin{array}{ll}
a_{1} & a_{2} \\
b_{1} & b_{2} \\
c_{1} & c_{2}
\end{array}\right]=\left[\begin{array}{rrrr}
1 & -1 & 1 & -1 \\
-1 & 1 & 1 & -1 \\
-1 & -1 & 1 & 1
\end{array}\right]\left[\begin{array}{ll}
x_{1} & y_{1} \\
x_{2} & y_{2} \\
x_{3} & y_{3} \\
x_{4} & y_{4}
\end{array}\right]
$$




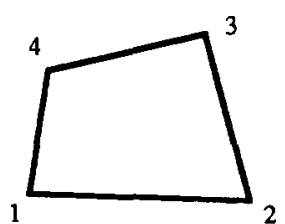

(a)

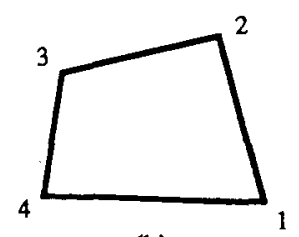

(b)

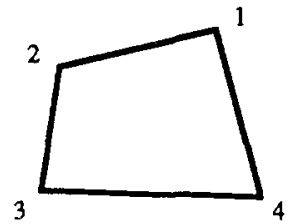

(c)

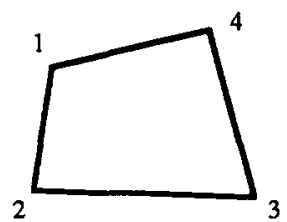

(d)

Fig. 2. Choices of a local node numbering scheme.

for the local node numbering scheme Fig. 2(a),

$$
\left[\begin{array}{ll}
a_{1} & a_{2} \\
b_{1} & b_{2} \\
c_{1} & c_{2}
\end{array}\right]=\left[\begin{array}{rrrr}
-1 & 1 & -1 & 1 \\
1 & 1 & -1 & -1 \\
-1 & 1 & 1 & -1
\end{array}\right]\left[\begin{array}{ll}
x_{1} & y_{1} \\
x_{2} & y_{2} \\
x_{3} & y_{3} \\
x_{4} & y_{4}
\end{array}\right]
$$

for the local node numbering scheme Fig. 2(b),

$$
\left[\begin{array}{ll}
a_{1} & a_{2} \\
b_{1} & b_{2} \\
c_{1} & c_{2}
\end{array}\right]=\left[\begin{array}{rrrr}
1 & -1 & 1 & -1 \\
1 & -1 & -1 & 1 \\
1 & 1 & -1 & -1
\end{array}\right]\left[\begin{array}{ll}
x_{1} & y_{1} \\
x_{2} & y_{2} \\
x_{3} & y_{3} \\
x_{4} & y_{4}
\end{array}\right]
$$

for the local node numbering scheme Fig. 2(c), and

$$
\left[\begin{array}{ll}
a_{1} & a_{2} \\
b_{1} & b_{2} \\
c_{1} & c_{2}
\end{array}\right]=\left[\begin{array}{rrrr}
-1 & 1 & -1 & 1 \\
-1 & -1 & 1 & 1 \\
1 & -1 & -1 & 1
\end{array}\right]\left[\begin{array}{ll}
x_{1} & y_{1} \\
x_{2} & y_{2} \\
x_{3} & y_{3} \\
x_{4} & y_{4}
\end{array}\right]
$$

for the local node numbering scheme Fig. 2(d).

A bilinear system of equations in the form of equation (7) cannot be solved in general. However, additional conditions can be provided by considering the geometric characteristics of quadrilateral isoparametric elements. These conditions are given by the following lemma.

Lemma. For any given quadrilateral isoparametric element, the following two inequalities must always hold:

$$
a_{1} \neq b_{1} \quad \text { and } \quad a_{2} \neq c_{2} \text {. }
$$

Proof. Let the given quadrilateral isoparametric element be locally numbered as shown in Fig. 2(a). Suppose $a_{1}=b_{1}$, then according to the explicit expressions for $a_{1}$ and $b_{1}$ in equation (9), $x_{1}$ must be equal to $x_{2}$. However, since the local nodes 1 and 2 in Fig. 2(a) will never have the same $x$-coordinates, $a_{1}=b_{1}$ can never be true. The same arguments concerning the $y$-coordinates can be made to prove $a_{2} \neq c_{2}$. By following the same logic, the lemma can be proved for local node numbering schemes Figs. 2(b), 2(c) and 2(d).

A compact notation to represent the determinant of a $2 \times 2$ matrix is introduced as

$$
r_{s}=\left|\begin{array}{ll}
r_{1} & s_{1} \\
r_{2} & s_{2}
\end{array}\right|=r_{1} s_{2}-r_{2} s_{1}
$$

where $r, s=a, b, c, d$. Notice that $r_{s}=-s_{r}$. 
Table 1

Solutions for inverse transformation analysis

\begin{tabular}{lll}
\hline No. & Condition & Solution \\
\hline 1 & $a_{1} a_{2} a_{b} a_{c} \neq 0$ & $a_{b} \xi^{2}+\left(c_{b}+d_{a}\right) \xi+d_{c}=0$, \\
2 & $a_{1}=0$ and $a_{2} c_{1} \neq 0$ & $\eta=\left(a_{d}+b_{a} \xi\right) / a_{c}$, \\
3 & $a_{2}=0$ and $a_{1} b_{2} \neq 0$ & where $\xi \in[-1.0,1.0]$ \\
4 & $a_{1} a_{2} \neq 0$ and $a_{b}=0$ & $\xi=\left(a_{1} d_{c}\right) /\left(b_{1} a_{c}+a_{1} a_{d}\right) ; \eta=a_{d} / a_{c}$ \\
5 & $a_{1} a_{2} \neq 0$ and $a_{c}=0$ & $\xi=a_{d} / a_{b} ; \eta=\left(a_{1} d_{b}\right) /\left(c_{1} a_{b}+a_{1} a_{d}\right)$ \\
6 & All other conditions & $\xi=d_{c} /\left(a_{1} d_{2}+b_{c}\right) ; \eta=b_{d} /\left(a_{2} d_{1}+b_{c}\right)$ \\
\hline
\end{tabular}

Theorem. There exists a unique inverse transformation for any quadrilateral isoparametric element. A complete set of general solutions to the inverse transformation is given by Table 1 .

Proof. The existence and uniqueness of solutions for the inverse transformation analysis are a consequence of the inverse function theorem [5] by the fact that the Jacobian determinant at every point in both the $\Omega_{\mathrm{e}}$ domain and the $\bar{\Omega}_{\mathrm{e}}$ domain is positive [8] and a one-to-one mapping relation exists [3]. To establish the solutions requires only elementary algebraic techniques. The derivation starts with one of the two possible values of $a_{1}$.

I. When $a_{1}=0$, the following cases may exist.

A. If $a_{2}=0$, then equation ( 7 ) reduces to a linear system

$$
\left[\begin{array}{ll}
b_{1} & c_{1} \\
b_{2} & c_{2}
\end{array}\right]\left\{\begin{array}{l}
\xi \\
\eta
\end{array}\right\}=\left\{\begin{array}{l}
d_{1} \\
d_{2}
\end{array}\right\} .
$$

From the existence and uniqueness condition, the determinant of the coefficient matrix in equation (15) must be nonzero and a unique solution is given by

$$
\xi=\frac{d_{1} c_{2}-d_{2} c_{1}}{b_{1} c_{2}-b_{2} c_{1}}, \quad \eta=\frac{b_{1} d_{2}-b_{2} d_{1}}{b_{1} c_{2}-b_{2} c_{1}} .
$$

This case satisfies the condition no. 6 in Table 1 . The solution from Table 1 is

$$
\begin{aligned}
& \xi=\frac{d_{c}}{a_{1} d_{2}+b_{c}}=\frac{d_{1} c_{2}-d_{2} c_{1}}{a_{1} d_{2}+b_{1} c_{2}-b_{2} c_{1}}=\frac{d_{1} c_{2}-d_{2} c_{1}}{b_{1} c_{2}-b_{2} c_{1}}, \\
& \eta=\frac{b_{d}}{a_{2} d_{1}+b_{c}}=\frac{b_{1} d_{2}-b_{2} d_{1}}{a_{2} d_{1}+b_{1} c_{2}-b_{2} c_{1}}=\frac{b_{1} d_{2}-b_{2} d_{1}}{b_{1} c_{2}-b_{2} c_{1}},
\end{aligned}
$$

which is identical to equation (16).

B. If $a_{2} \neq 0$, then equation (7) becomes

$$
\left[\begin{array}{ll}
b_{1} & c_{1} \\
b_{2} & c_{2}
\end{array}\right]\left\{\begin{array}{l}
\xi \\
\eta
\end{array}\right\}=\left\{\begin{array}{c}
d_{1} \\
d_{2}-a_{2} \xi \eta
\end{array}\right\} .
$$

It has been proved that $b_{1} \neq 0$ (Lemma). Therefore, only the value of $c_{1}$ needs to be considered to solve equation (17).

(a) If $c_{1}=0$, then a unique solution is obtained as

$$
\xi=\frac{d_{1}}{b_{1}}, \quad \eta=\frac{b_{1} d_{2}-b_{2} d_{1}}{a_{2} d_{1}+b_{1} c_{2}}
$$

This case also corresponds to condition no. 6 , and equation (18) can be deduced from Table 1. 
(b) If $c_{1} \neq 0$, then from the first equation of equation (17), $\eta$ may be expressed in terms of $\xi$. Substitution of $\eta$ into the second equation yields a quadratic equation of $\xi$ as

$$
a_{2} b_{1} \xi^{2}+\left(c_{2} b_{1}-a_{2} d_{1}-b_{2} c_{1}\right) \xi+\left(d_{2} c_{1}-c_{2} d_{1}\right)=0 \text {. }
$$

Two values of $\xi$ can be obtained by solving equation (19), but only the one which satisfies $\xi \in[-1.0,1.0]$ is the required unique solution. The value of $\eta$ is given by

$$
\eta=\frac{d_{1}-b_{1} \xi}{c_{1}} \text {. }
$$

It is easy to show that the solution given by equations (19) and (20) is the same as that obtained by condition no. 2 in Table 1.

II. When $a_{1} \neq 0$, the following cases may exist.

A. If $a_{2} \neq 0$, multiply the first equation of equation (7) by $a_{2}$ and the second one by $a_{1}$ and then subtract one from the other. Equation (7) becomes

$$
\left[\begin{array}{ll}
a_{b} & a_{c} \\
b_{1} & c_{1}
\end{array}\right]\left\{\begin{array}{l}
\xi \\
\eta
\end{array}\right\}=\left\{\begin{array}{c}
a_{d} \\
d_{1}-a_{1} \xi \eta
\end{array}\right\}
$$

(a) If $a_{b} \neq 0$, two cases arise depending on the value of $a_{c}$.

(1) If $a_{c} \neq 0$, solving equation (21) gives an equation for $\xi$ as

$$
a_{1} a_{b} \xi^{2}+\left(c_{1} a_{b}-a_{1} a_{d}-b_{1} a_{c}\right) \xi+\left(d_{1} a_{c}-c_{1} a_{d}\right)=0
$$

or

$$
a_{b} \xi^{2}+\left(c_{b}+d_{a}\right) \xi+d_{c}=0,
$$

where $\xi \in[-1.0,1.0]$, and an expression for $\eta$ as

$$
\eta=\frac{a_{d}-a_{b} \xi}{a_{c}}=\frac{a_{d}+b_{a} \xi}{a_{c}} .
$$

This solution is identical to that given in Table 1 by condition no. 1 .

(2) If $a_{c}=0$, the coefficients $a_{i}$ and $c_{i}(i=1,2)$ of equation (7) have a proportional relation, while $a_{i}$ and $b_{i}$ do not; that is,

$$
\frac{a_{1}}{a_{2}}=\frac{c_{1}}{c_{2}} \neq \frac{b_{1}}{b_{2}} \text {. }
$$

Therefore, a unique solution to equation (7) can be found. Since equation (21) now takes the form

$$
\left[\begin{array}{cc}
a_{b} & 0 \\
b_{1} & c_{1}
\end{array}\right]\left\{\begin{array}{l}
\xi \\
\eta
\end{array}\right\}=\left\{\begin{array}{c}
a_{d} \\
d_{1}-a_{1} \xi \eta
\end{array}\right\},
$$

the solution is

$$
\xi=\frac{a_{d}}{a_{b}}, \quad \eta=\frac{d_{1} a_{b}-b_{1} a_{d}}{c_{1} a_{b}+a_{1} a_{d}}=\frac{a_{1} d_{b}}{c_{1} a_{b}+a_{1} a_{d}},
$$

which is also given in Table 1 by condition no. 5 .

(b) If $a_{b}=0$, then $a_{c}$ must not be zero, because if $a_{c}=0$, then

$$
\frac{a_{1}}{a_{2}}=\frac{b_{1}}{b_{2}}=\frac{c_{1}}{c_{2}}
$$

which indicates that equation (7) has an infinite number of solutions, and that contradicts the uniqueness condition. Therefore,

$$
\frac{a_{1}}{a_{2}}=\frac{b_{1}}{b_{2}} \neq \frac{c_{1}}{c_{2}} \text {. }
$$


As in the case of II-A-(a)-(2), a unique solution can be found from the new form of equation (21)

$$
\left[\begin{array}{cc}
0 & a_{c} \\
b_{1} & c_{1}
\end{array}\right]\left\{\begin{array}{c}
\xi \\
\eta
\end{array}\right\}=\left\{\begin{array}{c}
a_{d} \\
d_{1}-a_{1} \xi \eta
\end{array}\right\}
$$

where

$$
\xi=\frac{d_{1} a_{c}-c_{1} a_{d}}{b_{1} a_{c}+a_{1} a_{d}}=\frac{a_{1} d_{c}}{b_{1} a_{c}+a_{1} a_{d}}, \quad \eta=\frac{a_{d}}{a_{c}} .
$$

This is the same solution as that given by condition no. 4 in Table 1 .

B. If $a_{2}=0$, then $c_{2} \neq 0$ by the Lemma, and equation (7) becomes

$$
\left[\begin{array}{ll}
b_{1} & c_{1} \\
b_{2} & c_{2}
\end{array}\right]\left\{\begin{array}{c}
\xi \\
\eta
\end{array}\right\}=\left\{\begin{array}{c}
d_{1}-a_{1} \xi \eta \\
d_{2}
\end{array}\right\} .
$$

This equation is similar to equation (17), case I-B, and the expression for a solution depends on the value of $b_{2}$.

(a) If $b_{2}=0$, then

$$
\xi=\frac{d_{1} c_{2}-c_{1} d_{2}}{a_{1} d_{2}+b_{1} c_{2}}=\frac{d_{c}}{a_{1} d_{2}+b_{1} c_{2}}, \quad \eta=\frac{d_{2}}{c_{2}},
$$

which can also be deduced from expression for condition no. 6 in Table 1.

(b) If $b_{2} \neq 0$, solving equation (31) gives the following for $\xi$ :

$$
a_{1} b_{2} \xi^{2}+\left(c_{1} b_{2}-a_{1} d_{2}-b_{1} c_{2}\right) \xi+\left(d_{1} c_{2}-c_{1} d_{2}\right)=0,
$$

where $\xi \in[-1.0,1.0]$, and the following for $\eta$ :

$$
\eta=\frac{d_{2}-b_{2} \xi}{c_{2}} \text {. }
$$

This case satisfies the condition no. 3 and the solution given by equations (33) and (34) can be obtained directly from Table 1 .

The above theorem gives a complete set of general solutions to the inverse transformation for any given quadrilateral isoparametric element. The above proof was conducted on a case-by-case basis to show the completeness of solutions and to provide an algorithm for computer implementation.

\section{Application for slope stability analysis}

Stress and strain evaluations in finite element analysis are usually conducted in terms of natural coordinates $(\xi, \eta)$ in the $\bar{\Omega}_{\mathrm{e}}$ domain. There are some circumstances in which the stress or strain evaluation is preferred to be performed in terms of global coordinates $(x, y)$ in the $\Omega_{\mathrm{e}}$ domain. Here slope stability analysis is used as an example.

Most of the methods used today for slope stability analysis fall into two approaches: limiting equilibrium methods (LEM) $[1,2,4]$ and finite element methods (FEM). For many practical engineering problems, a LEM is preferred for two reasons. First, it is easier to implement, and second, it provides civil engineers with a quantitative measure of slope stability in the form of a factor of safety against failure. Although the LEM can handle simple slopes, complex boundary conditions and unusual inclusions, such as soil reinforcement or underground structures, warrant a FEM for the analysis. 

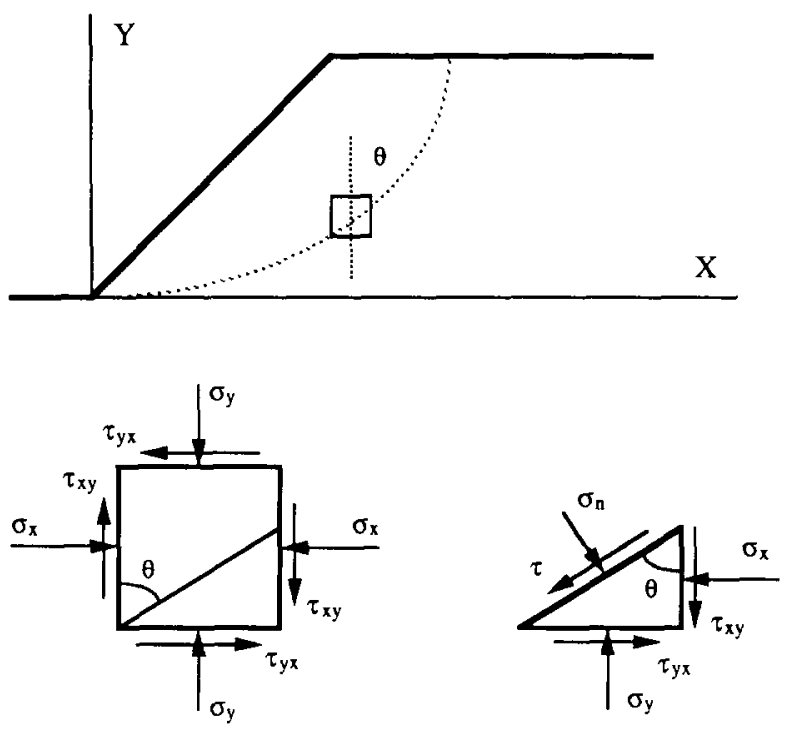

Fig. 3. Stress state at a point on an assumed failure surface.

On the other hand, although the FEM is very effective at providing stresses, strains and displacements, it does not furnish a factor of safety against failure. Therefore, neither LEM nor FEM alone is ideal for slope stability analysis. However, if the two methods are combined, a powerful approach to slope stability analysis can be developed. This is referred to as a FLEM (the combination of FEM and LEM) model for slope stability analysis.

The FLEM model consists of two procedures: (1) a FEM to obtain the stress field in a slope, and (2) a LEM to determine the minimum factor of safety against failure.

The stress state (either effective or total) at a point on an assumed failure surface is shown in Fig. 3. If the tangent to the failure surface at the point makes an angle $\theta$ with the vertical, the normal and shearing stresses on the surface at the point are given by

$$
\begin{aligned}
& \sigma_{n}=\frac{\sigma_{x}+\sigma_{y}}{2}+\frac{\sigma_{x}-\sigma_{y}}{2}+\tau_{x y} \sin 2 \theta, \\
& \tau=-\frac{\sigma_{x}-\sigma_{y}}{2} \sin 2 \theta+\tau_{x y} \cos 2 \theta .
\end{aligned}
$$

If the stresses along the failure surface can be computed from the finite element analysis, the normal and shearing stresses at every point on the failure surface can be computed by equations (35) and (36). The shearing strength, $s$, at any point on the surface can be obtained from the computed normal stress and Mohr-Coulomb theory [4], or

$$
s=c+\sigma_{\mathrm{n}} \tan \phi,
$$

where $c$ is the cohesion and $\phi$ is the internal angle of friction.

The total shearing strength and the total shearing force can then be found by summing the shearing strengths and shearing stresses at all points along the failure surface. Thus, a factor of safety against the surface failing can be defined as

$$
F S=\frac{\text { shearing strength on failure surface }}{\text { shearing force on failure surface }}=\frac{\sum_{i=1}^{N}\left(c_{i}+\sigma_{\mathrm{n} i} \tan \phi_{i}\right) \Delta L_{i}}{\sum_{i=1}^{N} \tau_{i} \Delta L_{i}},
$$


where $\Delta L_{i}$ is the incremental length at point $i$ along the failure surface and $N$ is the number of points on the surface at which stresses are evaluated. Different failure surfaces, such as planes, circles, logarithmic-spirals, parabolas, and so on, can then be assumed in search of an overall minimum factor of safety for the slope.

A problem in coupling FEM and LEM in FLEM is the determination of stresses along a failure surface. This problem results from the fact that a failure surface is defined in a $\Omega_{\mathrm{e}}$ domain while stress evaluation in FEM is usually done in the $\bar{\Omega}_{\mathrm{e}}$ domain. Two approaches were previously used to overcome this obstacle: either simple constant strain triangular elements were used which eliminated this problem because the stresses within such an element were constant, or, when quadrilateral isoparametric elements were used, the stresses along a given failure surface were interpolated from integration points. An obviously more effective and efficient solution would be to perform an inverse transformation analysis which would enable natural coordinates to be expressed in terms of global coordinates so that stress evaluation can be done directly in the element domain $\Omega_{\mathrm{e}}$.

\section{Conclusions}

The inverse transformation algorithm developed above allows for an explicit mapping of a point in the element domain $\Omega_{\mathrm{e}}$ into a parent domain $\bar{\Omega}_{\mathrm{e}}$. To some critical applications of finite elements, such as slope stability analysis, the inverse transformation provides an efficient approach for stress and strain evaluation in $\Omega_{\mathrm{c}}$.

\section{References}

[1] Bishop, A.W., "The use of slip circle in the stability analysis of slopes", Geotechnique 5 (1), pp. 7-17, 1955.

[2] JANBU, N., "Slope stability computation", in: Embankment-Dam Engineering, Casagrande Volume, edited by R.C. HiRsChfeld and S.J. Poulos, John Wiley \& Sons, New York, pp. 47-86, 1973.

[3] Kaplan, W., Advanced Mathematics for Engineers, Addison-Wesley, Reading, Massachusetts, p. 488, 1981.

[4] Lambe, T.W. and R.V. Whitman, Soil Mechanics, Wiley, New York, 1969.

[5] LANG, S., Real Analysis, Addison-Wesley, Reading, Massachusetts, 1969.

[6] TAIg, I.C., Structural Analysis by the Matrix Displacement Method, English Electric Aviation Report No. S017, 1961.

[7] Weaver, W., Jr. and P.R. Johnston, Structural Dynamics by Finite Elements, Prentice-Hall, Inc., Englewood Cliffs, New Jersey, p. 320, 1987

[8] Zienkiewicz, O.C. and R.L. TAYLoR, The Finite Element Method, 4th edn., Vol. 1, McGraw-Hill Book Company (UK) Limited, London, 1989. 